\title{
Pharmacodynamics and safety of lefradafiban, an oral platelet glycoprotein IIb/IIIa receptor antagonist, in patients with stable coronary artery disease undergoing elective angioplasty
}

K M Akkerhuis, M J B M van den Brand, C van der Zwaan, H O J Peels, H Suryapranata, L R van der Wieken, J Stibbe, J Hoffmann, T Baardman, J W Deckers, M L Simoons

\begin{abstract}
Objective-Lefradafiban is the orally active prodrug of fradafiban, a glycoprotein IIb/IIIa receptor antagonist. The present phase II study aimed to determine the dose of lefradafiban that provides $80 \%$ blockade of the glycoprotein IIb/IIIa receptors by fradafiban, and to study the pharmacodynamics and safety of different doses in patients with stable angina undergoing angioplasty.

Design-A double blind, placebo controlled, dose finding study.

Setting-Four academic and community hospitals in the Netherlands.

Patients-64 patients with stable coronary artery disease undergoing elective percutaneous transluminal coronary angioplasty.

Interventions-30 mg, $45 \mathrm{mg}$, and $60 \mathrm{mg}$ of lefradafiban three times daily or placebo was given for 48 hours.

Main outcome measures-The primary safety end point was the occurrence of bleeding, classified as major, minor, or insignificant according to the thrombolysis in myocardial infarction (TIMI) criteria. Efficacy indices included per cent fibrinogen receptor occupancy (FRO), ex vivo platelet aggregation, and plasma concentrations of fradafiban.

Results-Administration of lefradafiban 30, 45, and $60 \mathrm{mg}$ three times daily resulted in a dose dependent increase in median FRO levels of $71 \%, 85 \%$, and $88 \%$, respectively. Inhibition of platelet aggregation was closely related to FRO. There were no major bleeding events. The $60 \mathrm{mg}$ lefradafiban group had a high $(71 \%)$ incidence of minor and insignificant bleeding. The incidence of bleeding was $44 \%$ in the $30 \mathrm{mg}$ and $45 \mathrm{mg}$ groups, compared with $9 \%$ in placebo patients. Puncture site bleeding was the most common event. The odds of bleeding increased by $3 \%$ for every $1 \%$ increase in FRO.
\end{abstract}

Conclusions-Lefradafiban is an effective oral glycoprotein IIb/IIIa receptor blocker. The clinical effectiveness of doses up to $45 \mathrm{mg}$ three times daily should be investigated.

(Heart 2001;85:444-450)

Keywords: platelet aggregation inhibitors; glycoprotein IIb/IIIa blockers; lefradafiban; angioplasty

Platelet adhesion, activation, and aggregation are pivotal events in the process leading to coronary thrombosis in patients with unstable coronary artery disease. ${ }^{1}$ This process is also activated during percutaneous transluminal coronary angioplasty (PTCA) by local vascular injury, which exposes circulating platelets to various prothrombotic stimuli within the subendothelium. ${ }^{23}$ The final common pathway to coronary thrombus formation involves aggregation of platelets through cross linkage of their glycoprotein (GP) IIb/IIIa receptors by the primary binding ligand fibrinogen. ${ }^{4}$

Several inhibitors of the GP IIb/IIIa receptor have been developed. The monoclonal antibody c7E3 (abciximab) has been shown to reduce the incidence of death and myocardial infarction in patients undergoing PTCA, with or without stent deployment. ${ }^{5-8}$ Small molecules (eptifibatide, tirofiban) given intravenously also reduce complications associated with PTCA, although their effects were of borderline significance. ${ }^{910}$ These intravenous GP $\mathrm{IIb} / \mathrm{III}$ receptor blockers have also recently been shown to reduce the incidence of death, recurrent myocardial infarction, and recurrent ischaemia in patients with unstable angina or non-ST-segment elevation myocardial infarction. ${ }^{11-13}$

The clinical use of monoclonal antibodies and small molecules as GP IIb/IIIa receptor blockers is limited to the treatment of acute episodes of unstable angina, or treatment during coronary procedures, as oral administration is not feasible. Lefradafiban (Boehringer Ingelheim, Germany) is an orally active prodrug which is metabolised in two steps to fradafiban, an intravenously active, non-peptide GP IIb/ IIIa receptor inhibitor. ${ }^{14}$ In studies with healthy volunteers, both fradafiban and lefradafiban led to a reversible and dose dependent inhibition of platelet aggregation. ${ }^{14}$

Our aim in this first phase II study was to determine the dose regimen of lefradafiban that provides $80 \%$ blockade of the platelet GP $\mathrm{IIb} / \mathrm{III}$ a receptors by fradafiban, as well as to study the pharmacodynamics and safety of different doses in patients with stable coronary artery disease undergoing elective PTCA, in order to select the appropriate range of dose regimens for subsequent larger studies. 
Methods

STUDY POPULATION

Patients aged between 18 and 80 years with stable coronary artery disease were eligible for enrolment if they were scheduled for elective PTCA. Patients were excluded if they suffered from unstable angina or if there was a total occlusion of the vessel to be treated. Other criteria for exclusion included myocardial infarction within the preceding 14 days; planned stent implantation; major surgery or trauma within the preceding six weeks; any history of cerebrovascular haemorrhage or haemorrhagic diathesis, cardiopulmonary resuscitation, or complicated puncture of a major vein or artery within the preceding six weeks; retinopathy grade 3 or greater; gastrointestinal or genitourinary bleeding within the preceding six weeks; peptic ulcer disease; platelet count $<100 \times 10^{9} / 1$; uncontrolled hypertension (systolic blood pressure $>200 \mathrm{~mm} \mathrm{Hg}$ and/or diastolic blood pressure $>100 \mathrm{~mm} \mathrm{Hg}$ ); current treatment with oral anticoagulants or any antiplatelet agent other than acetylsalicylic acid, including any non-steroidal antiinflammatory agent; pregnant or nursing women or women not using medically approved means of contraception; known hepatic or renal insufficiency; or any other concomitant serious illness which would limit life expectancy or interfere with the study end points.

The study protocol was reviewed and approved by each hospital's institutional review board, and informed consent was obtained from each patient before participation in the trial.

STUDY DESIGN AND DOSE SELECTION

The initial protocol envisaged a dose escalation, starting with lefradafiban $60 \mathrm{mg}$ three times daily or placebo, and increasing to $75 \mathrm{mg}$ and $90 \mathrm{mg}$ three times daily. Based on phase I data in healthy volunteers, the latter dose was expected to achieve fibrinogen receptor occupancy (FRO) levels of more than $80 \% .{ }^{14}$ However, after studies on the first group of 21 patients treated with lefradafiban $60 \mathrm{mg}$ three times daily had been completed, it became apparent that this dose was associated with a high incidence of bleeding and with FRO levels of more than $80 \%$ (see Results). Therefore, the dose for the next group was reduced to $30 \mathrm{mg}$ three times daily. After the safety of this regimen had been established, a third group was investigated receiving lefradafiban $45 \mathrm{mg}$ three times daily or placebo.

Within each dose level, patients were randomised in a 4:1 ratio to receive lefradafiban or placebo in a double blind manner. The study drug was given as an oral solution three times a day for two days. It was not to be taken within two hours after or one hour before a meal. The first dose was given two hours before the start of the PTCA. An additional loading dose was given 3.5 hours after the first dose, while subsequent doses were given at eight hour intervals.
CONCOMITANT TREATMENT

Before the start of the PTCA, all patients received an intravenous bolus of heparin of $5000 \mathrm{IU}$, as well as an intravenous bolus of $250 \mathrm{mg}$ aspirin. During the procedure, heparin was repeated in boluses of 5000 IU after 30, 60 and, if necessary, 120 minutes. Aspirin was continued in a dose of $100 \mathrm{mg}$ daily. The use of ticlopidine together with lefradafiban was excluded by the protocol because no interaction data were available at that time. Therefore, if stenting was performed, the study drug was discontinued and ticlopidine $(250 \mathrm{mg}$ once or twice a day) was initiated. Other drugs were continued as before randomisation and adjusted as required by the clinical status of the patient.

CLINICAL AND LABORATORY MONITORING A coronary angiogram was performed immediately before and after PTCA. Coronary flow was classified according to the thrombolysis in myocardial infarction (TIMI) study classification by a central angiographic core laboratory (Cardialysis BV). ${ }^{15}$ Sheaths were removed 4-6 hours after discontinuation of the heparin infusion and as close as possible to the next intake of study drug, assuming that the platelet inhibitory activity of fradafiban would be at its nadir. Patients underwent daily clinical assessment for the occurrence of bleeding complications and other adverse events as well as extensive laboratory evaluation for haematology, coagulation, and biochemistry. They returned for a follow up visit two weeks after hospital discharge, which was not to take place within 48 hours from first study drug administration.

Samples for determination of FRO and plasma concentrations of fradafiban were obtained at baseline and at 2, 4, 12, 24, 36, and 48 hours after the first dose of the study drug. Blood for determination of FRO was stored in a Monovette prefilled with ACD (trisodium citrate, citric acid, dextrose). Platelet rich plasma was prepared by centrifugation and mixed with labelled fradafiban. After incubation and centrifugation, the supernatant as well as the platelet pellet were counted for free ligand in a $4 \mathrm{ml}$ scintillation cocktail. Nonspecific binding was determined in the presence of unlabelled fradafiban. The specific binding was calculated using corrections for spillover, extracellular space, and non-specific binding. The percentage of FRO was then calculated from the specific binding data, using corrections for receptor affinity and dilution of ACD.

Blood for determination of plasma fradafiban concentrations was drawn into tubes with EDTA. Plasma was separated immediately by centrifugation and stored at $-20^{\circ} \mathrm{C}$ until analysis. Plasma concentrations of fradafiban were measured using a validated high performance liquid chromatographic method with fluorescence detection. ${ }^{14}$ The lower limit of quantification was $1 \mathrm{ng} / \mathrm{ml}$.

Ex vivo platelet aggregation was assessed in a subgroup of 32 patients at baseline and at 2, 4, 24 , and 48 hours after study drug initiation. Platelet aggregation was measured in citrated 
platelet rich plasma and recorded by a Chrono-log aggregometer during a 6-8 minute interval after induction by two agonists: $1 \mathrm{mmol} / \mathrm{l} \mathrm{ADP}$ and $2 \mathrm{mg} / \mathrm{ml}$ collagen, respectively. Results are expressed as maximum per cent aggregation achieved in platelet rich plasma, using platelet poor plasma as reference of $100 \%$.

Ivy bleeding times determined with standard techniques were obtained at baseline and at the end of the 48 hour treatment period. Measurement of bleeding times was discontinued at 15 minutes.

\section{SAFETY AND EFFICACY INDICES}

The primary safety end point in this trial was the occurrence of bleeding complications, classified as major, minor, or insignificant according to the criteria of the TIMI study group. ${ }^{16}$ Major bleeding was defined as intracranial or bleeding associated with a decrease in haemoglobin of more than $3.1 \mathrm{mmol} / 1(5 \mathrm{~g} / \mathrm{dl})$ or a fall in haematocrit of more than $15 \%$. Minor bleeding was defined as observed blood loss with a decrease in haemoglobin of 1.9 $3.1 \mathrm{mmol} / 1(3.1-5.0 \mathrm{~g} / \mathrm{dl})$ or a fall in haematocrit of $10-15 \%$. Spontaneous gross haematuria or haematemesis, as well as a decrease in haemoglobin of $2.5-3.1 \mathrm{mmol} / 1$ or a fall in haematocrit of $12-15 \%$ without an identifiable bleeding site, were also considered to indicate minor bleeding. Blood loss insufficient to meet criteria for minor bleeding was classified as insignificant. Haemoglobin values were adjusted if patients received packed red blood cells or whole blood within 48 hours of the measurement. ${ }^{17}$

The primary efficacy variable was the FRO level in percentages, expressed as area under the curve normalised per hour. Secondary efficacy variables included the ex vivo platelet

Table 1 Clinical and angiographic characteristics at baseline

\begin{tabular}{|c|c|c|c|c|}
\hline & \multirow[b]{2}{*}{$\begin{array}{c}\text { Placebo } \\
(n=11)\end{array}$} & \multicolumn{3}{|c|}{ Lefradafiban dose ( $\times 3$ daily) } \\
\hline & & $\begin{array}{l}30 \mathrm{mg} \\
(\mathrm{n}=16)\end{array}$ & $\begin{array}{l}45 \mathrm{mg} \\
(\mathrm{n}=16)\end{array}$ & $\begin{array}{l}60 \mathrm{mg} \\
(\mathrm{n}=21)\end{array}$ \\
\hline \multicolumn{5}{|l|}{ Patient demographics } \\
\hline Age (years) (mean (SD)) & $58(7)$ & $65(8)$ & $62(13)$ & $60(12)$ \\
\hline Male sex (n) & 8 & 14 & 11 & 14 \\
\hline Diabetes (n) & - & 3 & 1 & 2 \\
\hline Hypertension (n) & 2 & 2 & 6 & 5 \\
\hline Previous MI (n) & 1 & 5 & 5 & 4 \\
\hline Previous PTCA or CABG (n) & 1 & 3 & 3 & 3 \\
\hline \multicolumn{5}{|l|}{ Angiographic variables } \\
\hline \multicolumn{5}{|l|}{ Segments to be treated (n) } \\
\hline RCA & 3 & 2 & 3 & 6 \\
\hline LAD & 6 & 9 & 8 & 8 \\
\hline LCX & 2 & 5 & 5 & 7 \\
\hline \multicolumn{5}{|l|}{ Perfusion grade, TIMI flow (n) } \\
\hline Grade 0 & 1 & - & 1 & - \\
\hline Grade 1 & 1 & - & - & 1 \\
\hline Grade 2 & 1 & 2 & 1 & 2 \\
\hline Grade 3 & 8 & 14 & 14 & 18 \\
\hline \multicolumn{5}{|l|}{ Lesion type by AHA/ACC (n) } \\
\hline A & 1 & - & 2 & 1 \\
\hline B1 & 5 & 3 & 5 & 4 \\
\hline B2 & 5 & 10 & 6 & 9 \\
\hline $\mathrm{C}$ & - & 3 & 3 & 5 \\
\hline Missing & - & - & - & 2 \\
\hline
\end{tabular}

AHA/ACC, American Heart Association/American College of Cardiology; CABG, coronary artery bypass grafting; LAD, left anterior descending artery; LCX, left circumflex artery; MI, myocardial infarction; PTCA, percutaneous transluminal coronary angioplasty; RCA, right coronary artery; TIMI, thrombolysis in myocardial infarction. aggregation and plasma concentrations of fradafiban.

STATISTICAL ANALYSIS

Descriptive analyses of angiographic, aggregation, and safety data are presented. The association of \% FRO, expressed as area under the curve (AUC), with the first bleeding event was assessed using logistic regression analysis, the null hypothesis being that the risk of bleeding was not affected by FRO levels. The AUC was calculated until the first bleeding, as well as over the whole treatment interval. The relation between bleeding and heparin administration as well as with the activated partial thromboplastin time (aPTT) AUC was investigated with logistic regression analysis in a similar way.

\section{Results}

Sixty four patients were enrolled: 16 received lefradafiban at a dose of $30 \mathrm{mg}$ three times daily, 16 received $45 \mathrm{mg}$ three times daily, 21 received $60 \mathrm{mg}$ three times daily, and 11 received placebo. The majority $(73 \%)$ of the patients were male (table 1). Their mean age was 61 years (range 39-79 years). A previous myocardial infarct was present in 15 patients $(23 \%)$, while three (5\%) had undergone coronary artery bypass surgery, and seven (11\%) had had PTCA. All patients received the study drug and underwent PTCA as scheduled. The treated segments were in the right coronary artery in 14 patients $(22 \%)$, in the circumflex coronary artery in $19(30 \%)$, and in the left anterior descending coronary artery in $31(48 \%)$. Coronary flow was normal (TIMI grade III) in 54 patients (84\%). Following PTCA, TIMI grade III flow was achieved in all patients, except two in the placebo group (TIMI 0), both caused by dissection. A stent was placed in nine patients. In these patients, the study drug was discontinued and ticlopidine (250 mg once or twice a day) was begun.

\section{PHARMACODYNAMICS AND INHIBITION OF} PLATELET AGGREGATION

Plasma concentrations of fradafiban increased in a dose dependent manner in patients treated with lefradafiban. Geometric means of the maximum plasma concentration of fradafiban at steady state were 158,314 , and $394 \mathrm{ng} / \mathrm{ml}$ during treatment with lefradafiban 30,45 , and $60 \mathrm{mg}$ three times daily, respectively (table 2 ). The pharmacokinetic profile of lefradafiban was predictable and no significant deviations from dose proportionality were observed.

There was a close correlation between the plasma concentrations of fradafiban and FRO (fig 1). FRO increased in a dose dependent manner in patients treated with lefradafiban. Median FRO was $0 \%$ in the placebo group, $71 \%$ in the lefradafiban $30 \mathrm{mg}$ group, $85 \%$ in the lefradafiban $45 \mathrm{mg}$ group, and $88 \%$ in the lefradafiban $60 \mathrm{mg}$ group (table 2). Minimum, mean, and maximum FRO values were also dose dependent. Plasma fradafiban concentrations of $170 \mathrm{ng} / \mathrm{ml}$ were required to achieve $80 \%$ FRO (fig 1). There was little variation in FRO within each patient (fig 2), while the 
Table 2 Efficacy and safety end points

\begin{tabular}{|c|c|c|c|c|}
\hline & \multirow[b]{2}{*}{$\begin{array}{l}\text { Placebo } \\
(n=11)\end{array}$} & \multicolumn{3}{|c|}{ Lefradafiban dose ( $\times 3$ daily) } \\
\hline & & $\begin{array}{l}30 \mathrm{mg} \\
(n=16)\end{array}$ & $\begin{array}{l}45 \mathrm{mg} \\
(n=16)\end{array}$ & $\begin{array}{l}60 \mathrm{mg} \\
(n=21)\end{array}$ \\
\hline \multicolumn{5}{|l|}{ Efficacy indices } \\
\hline Plasma concentration of fradafiban $(\mathrm{ng} / \mathrm{ml})^{\star}$ & 0 & $158 \quad(27)$ & $314 \quad(24)$ & $394 \quad(22)$ \\
\hline FRO $(\%)-$ minimum & 0 & 54 & 74 & 78 \\
\hline FRO $(\%)$-median & 0 & 71 & 85 & 88 \\
\hline FRO (\%)-maximum & 0 & 80 & 89 & 95 \\
\hline FRO $(\%)$-meant & 0 & 69 (12) & $83(6)$ & $87 \quad(5)$ \\
\hline \multicolumn{5}{|l|}{ Bleeding time } \\
\hline At baseline (min) & 3.3 & 4.0 & 4.1 & 5.3 \\
\hline After 48 hours (min) & 4.4 & 7.8 & 19.2 & 25.0 \\
\hline \multicolumn{5}{|l|}{ Bleeding complications } \\
\hline Patients with bleeding, $\mathrm{n}(\%)$ & $1(9)$ & $7 \quad(44)$ & $7 \quad(44)$ & $15(71)$ \\
\hline Number of bleeding events (n) & 1 & 8 & 9 & 29 \\
\hline \multicolumn{5}{|l|}{ Location of bleeding $(\mathrm{n})$} \\
\hline Vascular puncture site & 1 & 6 & 6 & 15 \\
\hline Gingival & - & 1 & 2 & 10 \\
\hline Skin & - & 1 & 1 & 2 \\
\hline Haematuria & - & - & - & 2 \\
\hline \multicolumn{5}{|l|}{ Severity of bleeding by TIMI (n) } \\
\hline Major & - & - & - & - \\
\hline Minor & - & - & 1 & 2 \\
\hline Insignificant & 1 & 8 & 8 & 27 \\
\hline
\end{tabular}

* Mean maximum fradafiban plasma concentration at steady state $\left(\mathrm{C}_{\mathrm{max} \times \mathrm{s}}\right)$ with the relative coefficient of variation (\%) in parentheses.

tRelative coefficient of variation (\%) in parentheses.

All FRO values are expressed as area under the curve normalised per hour. Relative coefficient of variation $=100 \times($ standard deviation $/$ mean $)$.

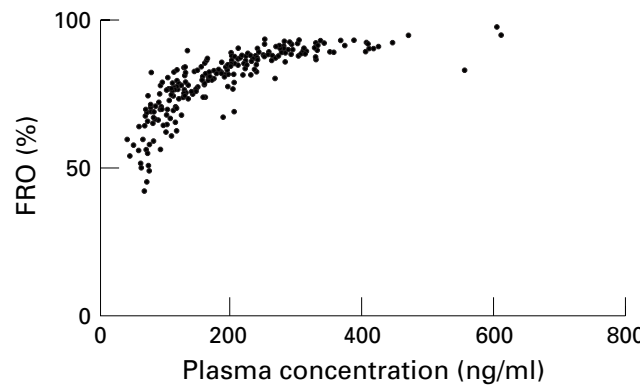

Figure 1 Relation between the plasma concentration of the active drug fradafiban and the degree of fibrinogen receptor occupancy (FRO).

interpatient variability was greater among patients receiving the lower dose than among those treated with the higher doses (fig 2 and table 2), reflecting the plasma concentrationFRO relation (fig 1).

Ex vivo platelet aggregation was measured in 32 patients and decreased with higher FRO levels (fig 3). In placebo patients, platelet aggregation was approximately $60 \%$ of normal. Inhibition of platelet aggregation to values below $20 \%$, or greater than $80 \%$ inhibition, was observed in all patients with FRO values above $80 \%$.

Bleeding times measured at 48 hours were in the normal range for all patients with FRO values below $70 \%$, while prolonged bleeding times were observed in the majority of patients with higher FRO values (fig 4).

\section{ADVERSE EVENTS}

Adverse events resulting in discontinuation of study drug treatment occurred in four patients: angina with cardiogenic shock in one $(30 \mathrm{mg})$, leucopenia in one $(30 \mathrm{mg})$, and bleeding in two other patients, both in the $45 \mathrm{mg}$ group. No major bleeding events were reported. Minor
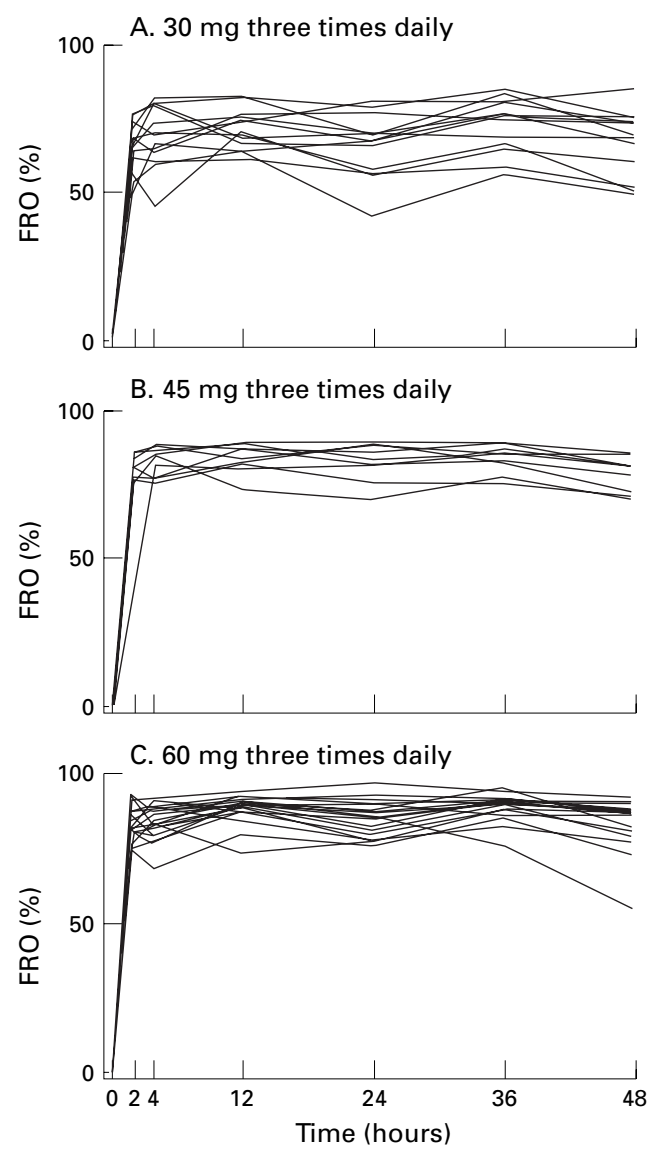

Figure 2 Degree of fibrinogen receptor occupancy (FRO) during oral administration of lefradafiban (A) $30 \mathrm{mg}$, (B) $45 \mathrm{mg}$, and (C) $60 \mathrm{mg}$ three times daily. Each line represents data from an individual patient in one of the three lefradafiban groups during 48 hours of oral treatment. Each line is based on FRO data obtained at one of the prespecified sample time points (baseline and 2, 4, 12, 24, 36 , and 48 hours after the study drug was started).

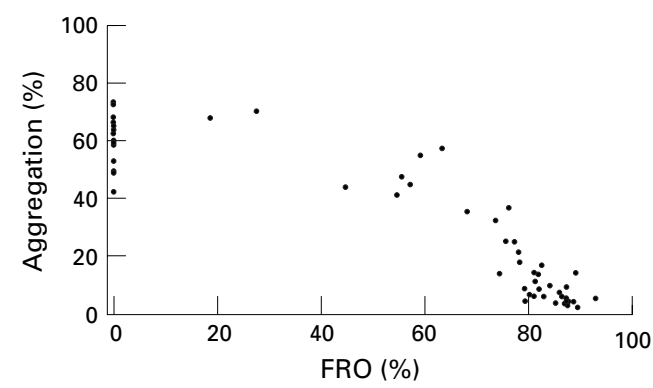

Figure 3 Relation between the degree of fibrinogen receptor occupancy (FRO) and inhibition of ex vivo platelet aggregation induced by $1 \mathrm{mmol} / \mathrm{l} A D P$ in platelet rich plasma. Each point represents data from a subject at a single time point during the 48 hours of oral treatment with lefradafiban 30, 45, or $60 \mathrm{mg}$ three times daily.

and insignificant bleeding complications were frequent in the highest dose group $(60 \mathrm{mg}$ three times a day), in which 15 of 21 patients (71\%) experienced such events (table 2). There was one haematoma in the placebo group, while bleeding was reported in seven $(44 \%)$ of 16 patients receiving lefradafiban $30 \mathrm{mg}$ three times daily, as well as in seven $(44 \%)$ of 16 patients receiving lefradafiban $45 \mathrm{mg}$ three times daily (table 2). Arterial and venous puncture site bleeding was most 


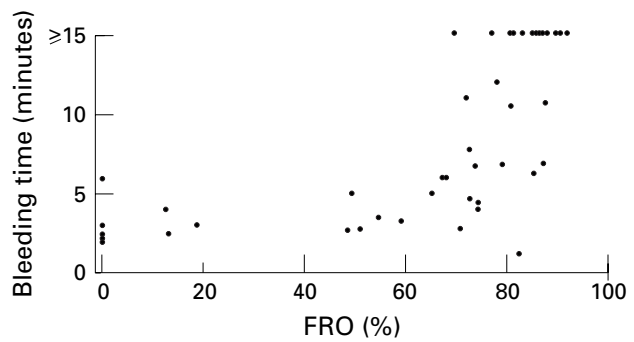

Figure 4 Relation between the degree of fibrinogen receptor occupancy (FRO) and ivy bleeding times at 48 hours after first study drug administration. Each point represents data from a subject in the lefradafiban 30,45, or $60 \mathrm{mg}$ group.

common. All bleeding events occurred more than two hours after administration of the first dose.

By logistic regression analysis, higher values of FRO were found to be significantly related to an increased incidence of bleeding. The odds of bleeding increased by a factor of 1.03 for every $1 \%$ increase in FRO $(\mathrm{p}<0.01)$. The relation between bleeding and heparin administration, as well as with the aPTT AUC, was explored in a similar way. When heparin administration and aPTT AUC were restricted to observations before the bleeding event, the relation with bleeding was significant for heparin $(p=0.04)$ but failed to reach conventional significance for aPTT $(p=0.08)$.

A clinically relevant fall in blood leucocyte count $\left(<3.0 \times 10^{9} / 1\right)$ was observed in three patients, two of whom were treated with lefradafiban $60 \mathrm{mg}$ three times daily, and one with lefradafiban $30 \mathrm{mg}$ three times daily. The lowest values were $0.9 \times 10^{9} / 1$ (from $5.9 \times 10^{9} / 1$ at baseline), $1.9 \times 10^{9} / 1$ (from $4.7 \times 10^{9} / 1$ ), and $2.3 \times 10^{9} / 1$ (from $\left.7.4 \times 10^{9} / 1\right)$, observed at 4,24 , and 48 hours after first study drug dose, respectively. After discontinuation of study drug, the leucocyte count in these patients returned to normal levels without additional measures within 2-5 days.

Median values for haematological variables at baseline were within normal limits in all treatment groups. There was a small decrease in haemoglobin and thrombocyte concentrations during the trial, which was comparable among all treatment groups. Median leucocyte count did not change. Serum concentrations of thromboplastin, thrombin, and fibrinogen were not affected. Median concentrations of biochemical variables at baseline were within the normal range in all treatment groups and remained within these limits at 24 and 48 hours.

\section{Discussion}

Administration of lefradafiban, an oral prodrug of the GP IIb/IIIa receptor blocker fradafiban, resulted in rapid and effective inhibition of platelet aggregation in patients with stable coronary artery disease undergoing elective PTCA. Doses of lefradafiban of 30, 45, and 60 mg three times daily caused a dose dependent increase in fradafiban plasma concentrations, an increase in FRO, and inhibition of platelet aggregation. With the three times daily dosing regimen, a stable FRO was achieved in each patient (fig 2). The level of inhibition achieved in patients who also received aspirin and heparin was greater than observed in volunteers receiving lefradafiban without additional drug treatment. In the placebo group, platelet aggregation was already decreased to $60 \%$. In the phase I studies, inhibition of platelet aggregation of more than $80 \%$ was achieved with lefradafiban doses of $90 \mathrm{mg}$ three times daily. ${ }^{14}$ In the present study, this was achieved with $45 \mathrm{mg}$ three times daily. This suggests an interaction between the GP IIb/IIIa receptor blocker and aspirin.

As in other studies with GP IIb/IIIa receptor blockers, bleeding occurred frequently at these levels of platelet inhibition and was dose dependent. The incidence of bleeding complications in the lefradafiban 30 and $45 \mathrm{mg}$ groups was similar to the rates observed in studies with other oral GP IIb/IIIa blockers at similar levels of platelet inhibition, ${ }^{18}{ }^{19}$ while even more bleeding occurred in the highest dose group $(71 \%)$. In studies with sibrafiban and xemilofiban, a gradual increase in bleeding was observed for higher dose levels with a greater degree of platelet inhibition. ${ }^{18} 19$ The results of the logistic regression analysis in the present study support these observations, with an increase in the risk of bleeding by $3 \%$ for every $1 \%$ increase in FRO. It should be appreciated that in the majority of cases the bleeding was insignificant, with a low incidence of minor bleeding complications (TIMI classification) and no major bleeding (table 2). Furthermore, all patients received aspirin and heparin and underwent invasive coronary procedures. Interactions between the GP IIb/IIIa receptor blocker abciximab and heparin have been reported in previous studies. ${ }^{56}$ As demonstrated in the EPILOG (evaluation of PTCA to improve long term outcome by c7E3 GP IIb/IIIa recptor blockade) and EPISTENT (evaluation of platelet GP IIb/IIIa inhibitor for stenting) studies, it is likely that a further reduction in the heparin dose in a weight adjusted manner might reduce bleeding complications in patients receiving lefradafiban without decreasing the clinical efficacy. ${ }^{78}$ Special care should be given to the access site of vascular sheaths. Sheaths should be removed early (4-6 hours) following the procedure, after the heparin has been stopped and the activated clotting time is 175 seconds or less. ${ }^{7}$ Given the interpatient variability in the degree of platelet inhibition observed with oral GP IIb/IIIa receptor antagonists, another potential strategy for reducing bleeding complications during longer term treatment could be to monitor the degree of platelet inhibition achieved in individual patients by using a platelet function bedside assay and to titrate the dose to a target level of inhibition. ${ }^{20} 21$

While a gradual dose dependent increase in bleeding was observed, as in studies with sibrafiban and xemilofiban, ${ }^{18}{ }^{19}$ the relation between bleeding time and per cent FRO in the present study suggests a threshold effect. The bleeding time was not increased at FRO values of less than $70 \%$, while a pronounced increase was observed at higher levels. It should be 
appreciated, however, that measurement of bleeding time does not reflect a tendency to spontaneous bleeding in clinical practice.

In three patients receiving lefradafiban, a clinically significant fall in blood leucocyte count was observed, although the mean leucocyte count did not change in the overall population. Leucopenia from bone marrow depression has been reported for another antiplatelet agent (ticlopidine). ${ }^{22}$ This, however, occurs mostly after 2-4 weeks of treatment. In the present study, leucopenia occurred immediately after the first dose or within two days. In all three patients, leucocytes returned rapidly in the peripheral blood after discontinuation of study drug. Leucopenia had not been observed in previous studies with lefradafiban in healthy volunteers and therefore was an unexpected finding in the present study. Following the observation of the leucopenia in the three patients, an amendment was made which increased the frequency of the haematological evaluations to monitor the safety of the patients and investigate the potential underlying pathophysiological mechanisms. However, no additional patients experienced this adverse event. A recently presented second phase II study with lefradafiban in patients with acute coronary syndromes has confirmed the increased incidence of leucopenia, or rather neutropenia, associated with lefradafiban, and has provided some insight into the pathophysiological mechanisms involved. ${ }^{23}$ In accordance with the present study, the decrease in neutrophils was characterised by early onset (immediately after first does of the study drug or within the next two days) and a rapid recovery after discontinuation of lefradafiban. Based on analysis of blood and bone marrow samples obtained in patients with neutropenia in that study, it was concluded that the observed neutropenia did not result from bone marrow depression but rather from a reversible redistribution of neutrophils by margination or clustering. More investigations are needed to clarify the precise mechanisms involved, as well as to determine the optimum duration of surveillance and the possible clinical associations.

The clinical benefit of treatment with GP IIb/IIIa receptor blockers in patients undergoing percutaneous intervention has been established by several studies using different inhibitors..$^{5-10}$ In the present limited series, all patients receiving lefradafiban had a successful PTCA without apparent thrombotic complications, while acute closure was observed during PTCA in one patient in the placebo group. Oral compounds such as lefradafiban offer an opportunity for long term treatment with an effective platelet aggregation inhibitor, which may enhance the early benefit of an intravenous agent and prevent subsequent thrombotic events in patients at risk.

Three recently presented large clinical trials have evaluated this concept of long term oral GP IIb/IIIa inhibition with xemilofiban in patients after percutaneous coronary intervention (evaluation of oral xemilofiban in controlling thrombotic events trial (EXCITE)) and with orbofiban and sibrafiban in patients after acute coronary syndromes (orbofiban in patients with unstable coronary syndromes trial (OPUS-TIMI-16), and sibrafiban versus aspirin to yield maximum protection from ischemic heart events post-acute coronary syndromes trial (SYMPHONY)). ${ }^{24-26}$ None of the trials showed a clear treatment benefit of long term GP IIb/IIIa inhibition, while all three trials showed a trend towards an increased mortality in the GP IIb/IIIa inhibitor group. ${ }^{24-26}$ In this respect, several points deserve consideration. First, the patient populations included in these trials were at relative low risk of recurrent thrombotic events. ${ }^{24-26}$ Furthermore, the pharmacokinetic and pharmacodynamic profile of these agents is characterised by a steep dose-response relation and by a short half life relative to the dosing interval. ${ }^{18} 1925$ This may have caused widely fluctuating inhibition of platelet aggregation and allowed complete recovery of platelet function between doses in some patients, with exposure of activated GP IIb/IIIa receptors on the platelet's surface. ${ }^{27}$ Additionally, recent studies have raised the possibility that platelet receptor antagonists may, at low concentrations, alter the steric conformation of the GP IIb/IIIa receptor sites and, paradoxically, enhance the thrombogenicity of these sites. ${ }^{27}{ }^{28}$ Compared with the other oral agents, the three times daily lefradafiban dosing regimen may result in higher average fradafiban concentrations with less peaktrough fluctuation and more stable levels of inhibition of platelet aggregation.

It is a challenge to exploit the potential beneficial antithrombotic effect of oral GP IIb/IIIa inhibitors in balance with the associated risk of haemorrhage. Dose titration to a target degree of platelet inhibition, measured with a rapid platelet function assay, may improve the overall safety and efficacy profile. ${ }^{2021}$ To increase the treatment benefit of (oral) GP IIb/IIIa blockers, one may choose to treat only patients who are at high risk of recurrent thrombotic complications, such as those who present with raised troponin concentrations and continue on medical treatment. These patients may gain particular benefit from a more aggressive long term therapeutic approach. ${ }^{29} 30$

Lefradafiban in doses up to $45 \mathrm{mg}$ three times daily achieved stable FRO values of more than $80 \%$ in patients with stable coronary artery disease undergoing elective PTCA, with acceptable safety during short term treatment. These doses may therefore be tested in further studies to assess whether long term treatment is indeed beneficial in patients with acute coronary syndromes at high risk of thrombotic complications, without an excess of bleeding complications.

We acknowledge the significant contribution of the following: Dr Rolf Brickl, Department of Pharmacokinetics and Drug Metabolism; Dr Brian Guth, Department of Pharmacology; Dr Gerhard Nehmiz and Suzanne Stolz, Department of Medical Data Services-Boehringer Ingelheim Pharma KG; Carlie Dille-Amo, Judith Rozendaal, Rene Stadhouders, Clemens Disco and Dr Gerrit-Anne van Es-Cardialysis BV, Clinical Research Management and Core Laboratories; Thea Muskee, Department of Haematology, University Hospital Rotterdam. We also wish to thank the local hospital personnel and the We also wish to thank the local hospital personnel and the
patients who agreed to participate. The study was supported by patients who agreed to
Boehringer Ingelheim. 
1 Fuster V, Badimon L, Badimon JJ, et al. The pathogenesis of coronary artery disease and the acute coronary syndromes.

2 Haerker LA. Role of platelets and thrombosis in mechanisms of acute occlusion and restenosis after angioplasty. Am $\mathcal{F}$ Cardiol 1987;60:20-8B.

3 Tenaglia AN, Fortin DF, Califf RM, et al. Predicting the risk of abrupt vessel closure after angioplasty in an individual patient. f Am Coll Cardiol 1994;24:1004-11.

4 Lefkovits J, Plow EF, Topol EJ. Platelet glycoprotein IIb/IIIa receptors in cardiovascular medicine. $N$ Engl $f \mathrm{Med}$ 1995;332:1553-9.

5 The EPIC Investigators. Use of a monoclonal antibody directed against the platelet glycoprotein IIb/IIIa receptor in high-risk coronary angioplasty. $N$ Engl f Med 1994;330: 956-61.

6 The CAPTURE Investigators. Randomised placebocontrolled trial of abciximab before and during coronary
intervention in refractory unstable angina: the CAPTURE study. Lancet 1997;349:1429-35.

7 The EPILOG Investigators. Platelet glycoprotein IIb/IIIa receptor blockade and low-dose heparin during percutanereceptor blockade and low-dose heparin during percutane1689-96.

8 The EPISTENT Investigators. Randomised placebocontrolled and balloon-angioplasty-controlled trial to assess safety of coronary stenting with use of platele glycoprotein-IIb/IIIa blockade. Lancet 1998;352:87-92.

9 The IMPACT-II Investigators. Randomised placebocontrolled trial of effect of eptifibatide on complications of percutaneous coronary intervention: IMPACT-II. Lancet 1997;349:1422-8

10 The RESTORE Investigators. Effects of platelet glycoprotein IIb/IIIa blockade with tirofiban on adverse cardiac
events in patients with unstable angina or acute myocardial infarction undergoing coronary angioplasty. Circulation 1997;96:1445-53.

11 The PRISM Study Investigators. A comparison of aspirin plus tirofiban with aspirin plus heparin for unstable angina. plus tirofiban with aspirin plus hepa

12 The PRISM-PLUS Study Investigators. Inhibition of the platelet glycoprotein IIb/IIIa receptor with tirofiban in unstable angina and non-Q-wave myocardial infarction. $N$ Engl f Med 1998;338:1488-97.

13 The PURSUIT Trial Investigators. Inhibition of platelet glycoprotein IIb/IIIa with eptifibatide in patients with acute coronary syndromes. $N$ Engl f Med 1998;339:436-43.

14 Müller TH, Weisenberger H, Brickl R, et al. Profound and sustained inhibition of platelet aggregation by fradafiban, nonpeptide platelet glycoprotein IIb/IIIa antagonist, and its orally active prodrug lefradafiban in men. Circulation 1997; 96:1130-8.

15 Chesebro J, Knatterud G, Roberts R, et al. Thrombolysis in myocardial infarction (TIMI) trial. Phase I: a comparison between intravenous plasminogen activator and intravenous streptokinase. Circulation 1987;76:142-54.

16 Rao AK, Pratt C, Berke A, et al. Thrombolysis in myocardial infarction (TIMI) trial-phase I: hemorrhagic manifestations and changes in plasma fibrinogen and the fibrinolytic system in patients treated with recombinant tissue plasminogen activator and streptokinase. 7 Am Coll Cardiol 1988;11:1-11.

17 Landefeld CS, McGuire E, Rosenblatt MW. A bleeding risk index for estimating the probability of major bleeding in hospitalized patients starting anticoagulant therapy. Am $\mathcal{F}$ Med 1990;89:569-78.

18 Kereiakes DJ, Kleiman NS, Ferguson JJ, et al, for the ORBIT Trial Investigators. Pharmacodynamic efficacy, clinical safety, and outcomes after prolonged platelet glycoprotein IIb/IIIa receptor blockade with oral xemilofiban: results of a multicenter, placebo-controlled, randomized trial. Circulation 1998:98:1268-78.

19 Cannon CP, McCabe CH, Borzak S, et al, for the TIMI 12 Investigators. Randomized trial of an oral platelet glycoprotein IIb/IIIa antagonist, sibrafiban, in patients after an acute coronary syndrome: results of the TIMI 12 trial. Circulation 1998;97:340-9.

20 Coller BS. Monitoring platelet GP IIb/IIIa antagonist therapy. Circulation 1998;97:5-9.

21 Smith JW, Steinhubl SR, Lincoff AM, et al. Rapid platelet-function assay: an automated and quantitative cartridge-based method. Circulation 1999;99:620-5.

22 Yeh SP, Hsueh EJ, Wu H, et al. Ticlopidine-associated aplastic anemia. A case report and review of literature. Ann Hematol 1998;76:87-90.

23 Wilcox RG for the FROST Investigators. Preliminary results of the fibrinogen receptor occupancy study (FROST) Congress of the American College of Cardiology, March 7-10, 1999 .

24 O'Neill WW for the EXCITE Investigators. Results of the evaluation of oral xemilofiban in controlling thrombotic events (EXCITE) trial. Congress of the American College of Cardiology, March 7-10, 1999.

25 Cannon CP for the OPUS-TIMI-16 Investigators. Preliminary results of the TIMI-16 orbofiban in patients with unstable coronary syndromes (OPUS) trial. Congress of the American College of Cardiology, March 7-10, 1999.

26 The SYMPHONY Investigators. Comparison of sibrafiban with aspirin for prevention of cardiovascular events after acute coronary syndromes: a randomized trial. Lancet 2000;355:337-45.

27 Peter K, Straub A, Kohler B, et al. Platelet activation as a potential mechanism of GP IIb/IIIa inhibitor-induced thrombocytopenia. Am f Cardiol 1999;84:519-24.

28 Peter K, Schwarz M, Ylanne J, et al. Induction of fibrinogen binding and platelet aggregation as a potential intrinsic property of various glycoprotein $\mathrm{IIb} / \mathrm{IIIa}\left(\alpha_{\mathrm{II}} \beta_{3}\right)$ inhibitors. Blood 1998;92:3240-9.

29 Hamm CW, Heeschen C, Goldmann B, et al. for the CAPTURE Investigators. Benefit of abciximab in patients with refractory unstable angina in relation to serum troponin $\mathrm{T}$ levels. N Engl f Med 1999;340:1623-9.

30 Heeschen C, Hamm CW, Goldmann B, et al, for the PRISM Study Investigators. Troponin concentrations for stratification of patients with acute coronary syndromes in relation to of patients with acute coronary syndromes in relation 62 .

\section{Electronic pages}

\section{eHEART: www.heartjnl.com}

The following electronic only articles are published in conjunction with this issue of Heart (see also p 443).

\section{Behçet's disease with a large intracardiac thrombus: a case report}

M Baykan, Ş Çelik, C Erdöl, E C Baykan, I Durmuş, $S$ Bahadir, $H$ Erdöl, $C$ Örem, $H$ Çakirbay

Behçet's disease is recognised as a chronic multisystem disorder with vasculitis as its underlying pathological process. Cardiac involvement is rare and often associated with poor prognosis. A case of a 33 year old man with Behçet's disease, presenting with a large right ventricle and right atrial thrombus, is reported. Two dimensional (cross sectional), colour Doppler, and transoesophageal echocardiography, angiography, computed tomography, and magnetic resonance imaging were used to diagnose the disease. With cyclophosphamide and dexamethasone treatment, the cardiac lesions progressively resolved.

(Heart 2001;85:e7) www.heartjnl.com/cgi/content/full/85/ $4 / \mathrm{e} 7$
Serious adverse events experienced by patients with chronic heart failure taking spironolactone $C$ Berry, $\mathcal{F}$ f V McMurray

In patients with chronic heart failure, spironolactone added to conventional treatment may lead to serious and, occasionally, fatal hyperkalaemia. In some cases this seems to happen because spironolactone causes diarrhoea. Four cases involving men with New York Heart Association functional class III heart failure are presented. As these cases revealed, close monitoring of blood chemistry is mandatory after starting spironolactone, and patients should be advised to stop spironolactone immediately if diarrhoea develops.

(Heart 2001;85:e8) www.heartjnl.com/cgi/content/full/85/ $4 / \mathrm{e} 8$ 